Revista de Matemática: Teoría y Aplicaciones 3(1): 27-34 (1996)

\title{
SUBMARTINGALAS FINITAS, TEOREMAS DE CONVERGENCIA Y MÉTODOS NO STANDARD
}

\author{
Jaime Lobo SegurA ${ }^{1}$
}

\begin{abstract}
Resumen
Se examinan dos teoremas clásicos de convergencia de submartingalas en tiempo discreto, los de convergencia casi segura y en $\mathcal{L}^{1}$, usando los métodos del análisis no standard. Se realiza para ello un estudio previo sobre las submartingalas finitas. El marco formal adoptado para el análisis no standard es el de la "Internal Set Theory" de E. Nelson.
\end{abstract}

Con la introducción de los métodos no standard en la teoría de probabilidades se ha hecho patente el interés de las sucesiones de variables aleatorias finitamente indexadas, es decir de las variables aleatorias indexadas por un intervalo finito de $\mathbb{N}$. Para abreviar, en este trabajo las llamaremos sucesiones aleatorias finitas, o bien sucesiones finitas, y su definición precisa aparece más adelante.

Ciertos autores han puesto de manifiesto el interés de las sucesiones finitas para el estudio de los problemas de convergencia de sucesiones aleatorias clásicas. Estas son usadas, aunque no explícitamente, en el trabajo de Helms y Loeb [5], donde se establecen los teoremas de convergencia de Doob para submartingalas. En este trabajo no se realiza sin embargo un estudio independiente para las sucesiones finitas. Por otro lado E. Nelson desarrolla en [7] una teoría autocontenida de sucesiones finitas en espacios de probabilidad finitos y ofrece algunos resultados (cap. 11 y 12) sobre convergencia de submartingalas finitas. Sin embargo no parece posible a partir de los resultados de Nelson, y aun usando los teoremas de equivalencia en [7], deducir los teoremas clásicos antes mencionados.

En este trabajo presento una prueba de los teoremas de Doob por medio de un estudio sistemático de las submartingalas finitas. Se completa de esta manera los resultados de Nelson y se simplifica la prueba de Helms y Loeb. Se adopta para esto la versión de la Internal Set Theory (IST) del análisis no standard. Se presentará primeramente en la sección 1 los resultados de convergencia del tipo no standard para submartingalas finitas. De estos y de un teorema de equivalencia sobre las convergencias no standard y clásicas se deduce, en la sección 2 , los teoremas clásicos.

Las sucesiones finitas se denotarán por $\left(X_{n}\right)_{n \leq \mu}$, siendo en general $\mu$ un entero ilimitado. Una sucesión finita es submartingala (resp. martingala) finita con respecto a una filtración finitamente indexada $F=\left(F_{n}\right)_{n \leq \mu}$ si es integrable, $F$-adaptada y cumple la

\footnotetext{
${ }^{1}$ Escuela de Matemática, Universidad de Costa Rica, 2060 San José, Costa Rica
} 
condición $E_{n}\left(X_{n+1}\right) \geq X_{n}$ (resp. $\left.E_{n}\left(X_{n+1}\right)=X_{n}\right)$ para $n \leq \mu-1,\left(E_{n}()\right.$ denota la esperanza condicional con respecto a la tribu $F_{n}$ de $F$ ). Dada una sucesión aleatoria $X=\left(X_{n}\right)_{n \in \mathbb{N}}$ se define para cada $\mu$ entero la sucesión finita $X^{\mu}=\left(X_{n}\right)_{n \leq \mu}$, llamada la sucesión $X$ truncada en $\mu$. Si la sucesión $X$ es además submartingala (resp. martingala) con respecto a una filtración $F$, la sucesión truncada a $X^{\mu}$ es a su vez submartingala (resp. martingala) con respecto a la filtración $F$ truncada en $\mu$.

Se hará uso de la terminología y notación no standard siguientes: un número real es infinitesimal si $|x| \leq a$ para todo $a>0$ standard. Es llamado ilimitado si $|x|>a$ para todo real $a$ standard. Si $x, y$ son reales denotamos: $x \approx y$ si $x-y$ es infinitesimal, $x \approx \infty$ si $x$ es ilimitado positivo, $x \ll \infty$ si $x$ no es ilimitado positivo. Sea $\mu$ un entero no standard. Se dice que una sucesión finita $\left(X_{n}\right)_{n \leq \mu}$ converge n.s. (converge "nearly surely") si para todo $\gamma \approx \infty, \gamma \leq \mu \mathrm{y} \epsilon \gg 0$ :

$$
P\left(\max _{\gamma \leq i \leq \mu}\left|X_{i}-X_{\mu}\right|>\epsilon\right) \approx 0
$$

Dado $p \geq 1$, una sucesión finita de variables $p$-integrables $\left(X_{n}\right)_{n \leq \mu}$ converge n.s. en $\mathcal{L}^{p}$ si para todo $\gamma \approx \infty, \gamma \leq \mu$ se tiene $\left\|X_{\gamma}-X_{\mu}\right\|_{p} \approx 0$. Decimos que una variable $x$ es $S L^{1}$ (o Loeb-Nelson integrable) si para todo $a \approx \infty$ se tiene $E\left(|x|^{1}\{|x|>a\}\right) \approx 0$.

\section{Convergencia de submartingalas finitas}

Usaremos las desigualdades siguientes de Doob ([4]): siendo $\left(X_{n}\right)_{n \leq \mu}$ una submartingala o supermartingala con respecto a una filtración finita, entonces para todo $\lambda>0$ :

(1): $P\left(\max _{p, p \leq \mu}\left|X_{p}-X_{0}\right|>\lambda\right) \leq 2 / \lambda|| X_{\mu}-X_{0} \|_{1}$

(2): $P\left(\max _{p, p \leq \mu} X_{p}>\lambda\right) \leq 1 / \lambda\left\|X_{0}\right\|_{1}$,

si $X$ es supermartingala positiva.

Es de notar que estas desigualdades del tipo Doob pueden ser obtenidas sin recurrir a los llamados teoremas de paro ("stopping times") (ver [7] para la desigualdad (1) y [5] para la (2)).

Se establecen primero algunos lemas preliminares. En ellos se supone dada una filtración finitamente indexada con respecto a la cual se cumplen las propiedades de submartingala.

Lema 1 Sea $X=\left(X_{n}\right)_{n \leq \mu}$ una submartingala positiva finita tal que la sucesión numérica finita: $\left(E\left(X_{n}^{2}\right)\right)_{n \leq \mu}$ n.s. converge. Entonces $X$ converge n.s. en la métrica $\mathcal{L}^{2}$ y también n.s. converge.

Demostración: Sea $\mu \geq m>n$. Entonces:

$$
E\left(\left(X_{n}-X_{m}\right)^{2}\right)=E\left(X_{n}^{2}\right)-2 E\left(X_{n} X_{m}\right)+E\left(X_{m}^{2}\right) \leq E\left(X_{m}^{2}\right)-E\left(X_{n}^{2}\right)
$$

donde la desigualdad se obtiene por ser $X$ una submartingala positiva. La convergencia n.s. de la sucesión numérica implica entonces que para $m, n \approx \infty$ se tiene $E\left(X_{m}^{2}\right)-E\left(X_{n}^{2}\right) \approx 0$, 
y por lo tanto $E\left(\left(X_{n}-X_{m}\right) 2\right) \approx 0$, que expresa la convergencia n.s. en $\mathcal{L}^{2}$ de $X$. La convergencia n.s. es una consecuencia de la desigualdad (1) y de la desigualdad $\|\cdot\|_{1} \leq\|\cdot\|_{2}$.

Lema 2 Sea $X=\left(X_{n}\right)_{n \leq \mu}$ una submartingala finita. Entonces $X$ se descompone como $X=M-N$, siendo $M$ martingala positiva y $N$ supermartingala positiva. Además $E\left(M_{0}\right)=E\left(X_{\mu}^{+}\right)$.

Demostración: Definimos la martingala $M$ como $M_{n}=E_{n}\left(X_{\mu}^{+}\right)$, donde $X_{\mu}^{+}$denota como es usual la parte positiva de $X_{\mu}$, y es evidentemente positiva. Además $N_{n}=M_{n}-X_{n}$ es una supermartingala positiva (martingala positiva si $X$ es martingala).

En efecto, como $\left(X_{n}^{+}\right)_{n \leq \mu}$ es una submartingala, se tiene $M_{n} \geq X_{n}^{+}$; por lo tanto $M_{n}-$ $X_{n}=M_{n}-X_{n}^{+}+X_{n}^{-} \geq 0$, lo que ofrece la descomposición buscada.

Para el resultado que sigue usaremos el principio sucesional: si una sucesión numérica finita $a_{1}, \ldots, a_{\mu}$ es creciente y acotada por un real limitado entonces existe $\nu \approx \infty$ menor que $\mu$ tal que $a_{1}, \ldots, a_{\nu}$ converge n.s. Este resultado es un corolario inmediato de un resultado más general (teorema 6.1, [7]) sobre sucesiones de "fluctuación limitada".

Proposición 1 Sea $X=\left(X_{n}\right)_{n \leq \mu}$ una supermartingala finita positiva que cumple $E\left(X_{0}\right) \ll \infty$. Existe entonces $\nu \approx \infty$ menor que $\mu$ tal que el proceso truncado $X^{\nu}$ converge n.s.

Demostración: Siendo $-X$ una submartingala negativa y la función exponencial una función convexa y creciente, la sucesión finita $\mathbf{X}=\exp (-X)$ así como $\mathbf{X}^{2}$ son submartingalas finitas positivas acotadas por 1 .

La sucesión numérica finita $m=\left(E\left(\mathbf{X}_{n}^{2}\right)\right)_{n \leq \mu}$ es pues creciente, acotada por 1. Gracias al principio sucesional existe $\nu \approx \infty$ menor que $\mu$ tal que la sucesión truncada $m^{\nu}$ converge n.s. Del lema 1 la submartingala truncada $\mathbf{X}^{\nu}$ converge n.s.

Se desea probar ahora que el entero $\nu$ ilimitado es el conveniente, es decir que $X^{\nu}$ converge n.s. Dado $\epsilon \gg 0$ y $\gamma$ entero ilimitado menor que $\nu$ definimos el evento:

$$
A_{\gamma, \nu}^{\epsilon}=\left\{\max _{\gamma \leq i \leq \nu}\left|X_{i}-X_{\nu}\right|>\epsilon\right\}
$$

Entonces para todo $\lambda>0$ :

$$
P\left(A_{\gamma, \nu}^{\epsilon}\right)=P\left(A_{\gamma, \nu}^{\epsilon} \mid\left\{\max _{p \leq n} X_{p}>l\right\}\right)+P\left(A_{\gamma, \nu}^{\epsilon} \mid\left\{\max _{p \leq n} X_{p} \leq l\right\}\right)
$$

Sea $\delta \gg 0$ dado. El primer término en la última expresión es menor que $P\left(\left\{\max _{p \leq \nu} X_{p}>\right.\right.$ $\lambda\})$ y éste, aplicando la desigualdad (2) a $X^{\nu}$, es a su vez menor que $\delta / 2$ para un $\lambda \ll \infty$ adecuado. El segundo término es infinitesimal para todo $\lambda \ll \infty$. En efecto, de la desigualdad:

$$
\left|e^{-x}-e^{-y}\right| \geq|x-y| e^{-\lambda} \text { si } x, y \in[0, \lambda]
$$

este término es menor que $P\left(\max _{\gamma \leq i \leq \nu}\left|\mathbf{X}_{i}-\mathbf{X}_{\nu}\right|>\epsilon e^{-\lambda}\right)$ y es infinitesimal para todo $\lambda \ll \infty$ pues $\epsilon e^{-\lambda} \gg 0$ y del hecho de que la submartingala $\mathbf{X}^{\nu}$ es n.s. convergente. 
Por lo tanto $P\left(A_{\gamma, \nu}^{\epsilon}\right) \leq \delta$ y siendo $\delta \gg 0$ arbitrario se deduce que $P\left(A_{\gamma, \nu}^{\epsilon}\right) \approx 0$. Esto es cierto para todo $\epsilon \gg 0$ y $\gamma$ entero ilimitado menor que $\nu$, lo que implica que $X^{\nu}$ es n.s. convergente.

Se obtienen así las condiciones suficientes de convergencia n.s. para submartingalas finitas:

Teorema 1 Sea $X=\left(X_{n}\right)_{n \leq \mu}$ una submartingala finita que cumple $E\left(\left(X_{\mu}\right)^{+}\right) \ll \infty y$ $E\left(\left|X_{0}\right|\right) \ll \infty$. Existe un entero $\nu$ ilimitado menor que $\mu$ tal que $X^{\nu}$ converge n.s.

Demostración: Sea $X=M-N$, la descomposición de $X$ obtenida en el lema 2. Tanto $M$ como $N$ son supermartingalas finitas y $E\left(M_{0}\right)=E\left(\left(X_{\mu}\right)^{+}\right) \ll \infty, E\left(N_{0}\right)=$ $E\left(M_{0}\right)-E\left(X_{0}\right)<<\infty$. Se cumplen entonces las condiciones de la proposición 1 , tanto para $M$ como para $N$. Es fácil ver que se puede escoger $\nu$ entero ilimitado menor que $\mu$ tal que los procesos truncados $M^{\nu}$ y $N^{\nu}$ convergen ambos n.s. Entonces $X^{\nu}=M^{\nu}-N^{\nu}$ converge también n.s.

\section{Convergencia de submartingalas en el sentido clásico}

Para deducir los resultados de convergencia para sucesiones aleatorias necesitamos la equivalencia entre convergencias del tipo clásico y las no standard. Esto se enuncia en la proposición:

Proposición 2 Sea una sucesión standard $X$ de variables $\left(X_{n}\right)_{n \in \mathbb{N}}$ definidas en espacio standard $(\Omega, \mathcal{A}, P)$.

(i) $X$ es casi siempre de Cauchy solo si para todo $\mu \approx \infty X^{\mu}$ converge n.s.

(ii) Si para algún $\mu \approx \infty X^{\mu}$ converge n.s. entonces $X$ es casi siempre de Cauchy.

(iii) $X$ es de Cauchy en la norma $\mathcal{L}^{1}$ si y solo si para todo $\mu \approx \infty, X^{\mu}$ converge n.s en $\mathcal{L}^{1}$.

(iv) Si $X$ es equiintegrable entonces $X_{\mu}$ es $S L^{1}$ para todo $\mu$, con $\mu \approx \infty$.

El resultado (iii) se prueba de la misma manera que para la caracterización no standard de las sucesiones de Cauchy reales standard. En cuanto a (i) y (ii), Helms y Loeb enuncian en [5] un resultado cercano omitiendo su prueba (en [5] no se hace por lo demás uso de este hecho). En este mismo trabajo se menciona la condición necesaria en (iv) sin establecer su relación con la equiintegrabilidad. Por su importancia daremos en el apéndice una prueba de estas equivalencias según el enfoque de la IST.

Podemos ahora presentar una prueba no standard de los teoremas de convergencia en el sentido casi seguro y en $\mathcal{L}^{1}$. En un espacio de probabilidades $(\Omega, A, P)$ provisto de una filtración $F=\left(F_{n}\right)_{n \in \mathbb{N}}$ se da una submartingala en tiempo discreto $X=\left(X_{n}\right)_{n \in \mathbb{N}}$ adaptada a $F$. Entonces tenemos (Doob, [4]): 
(i) Si la condición: $\sup _{n \in \mathbb{N}} E\left(X_{n}^{+}\right)<\infty$ se cumple, $X$ converge casi siempre a un límite finito.

(ii) Si $X$ es equiintegrable, converge casi siempre y en $\mathcal{L}^{1}$.

Prueba no Standard: Tanto en (i) como en (ii) basta por transfert suponer que $(\Omega, A, P), F$ y $X$ son standard.

Bajo las condiciones de (i) la sucesión $m=\left(E\left(X_{n}^{+}\right)\right)_{n \in \mathbb{N}}$ es entonces standard y convergente.

La caracterización no standard de las sucesiones convergentes dice que $E\left(X_{\mu}^{+}\right) \ll \infty$ para todo $\mu$ entero ilimitado. El valor de $E\left(\left|X_{0}\right|\right)$ es standard y por lo tanto limitado.

Entonces para un tal entero $\mu$ la submartingala finita $X^{\mu}$ cumple las condiciones del teorema 1 , y por lo tanto existe $\nu$ entero ilimitado menor que $\mu$ tal que $X^{\nu}$ converge n.s. Por (ii) de la proposición 2, $X$ es casi siempre de Cauchy, lo que establece (i).

Las condiciones de (ii) siendo más fuertes que en (i), de lo anterior se obtiene el resultado de convergencia c.s. de (ii).

Por (i) de prop. $2 X^{\mu}$ converge n.s. para todo $\mu$ entero ilimitado. Sea $\gamma$ ilimitado menor que $\mu$. La propiedad de equiintegrabilidad implica ((iv) de prop. 2) que $X_{\mu}, X_{\gamma}$ son $S L^{1}$ y como $X_{\mu} \approx X_{\gamma}$ n.s., de la versión $S L^{1}$ del teorema de Lebesgue (ver [7], cap. 8), obtenemos que $\left\|X_{\mu}-X_{\gamma}\right\|_{1} \approx 0$.

Empleando de nuevo la prop. 2 obtenemos que $X$ converge en $\mathcal{L}^{1}$.

\section{Observaciones finales}

1. Un argumento importante usado en la sección 1 es el principio sucesional para la prueba de la proposición 1. Este es una consecuencia del axioma de standardización de la IST (ver [6] o bien la discusión muy completa efectuada en [3], cap.3). Sería interesante buscar un argumento más sencillo que no dependa de este principio, pues se ha señalado que los métodos de la IST pueden prescindir de dicho axioma.

2. Un resultado cercano al obtenido en el teorema 1 en el marco de espacios finitos es dado por Nelson en [7] (teorema 12.3). En efecto, bajo las condiciones del teorema 1, del teorema de Nelson y del principio sucesional se deduce que la propiedad externa "existe $n$ ilimitado tal que $X^{\nu}$ converge" se cumple casi siempre. Es un resultado menos fuerte que el del teorema 1 pues este último se puede expresar como: existe $\nu$ ilimitado tal que la propiedad externa " $X^{\nu}$ converge" se cumple casi siempre.

3. Algunos resultados de la sección 2 se inspiran en pruebas clásicas presentadas por diferentes autores. Una versión clásica del lema 1 se encuentra en [2], y el lema 2 es una versión para sucesiones finitas de la llamada descomposición de Krickeberg (ver [2] o bien [8]).

4. Los métodos no standard han sido también abordados en [1] para establecer un resultado muy general sobre problemas límite en análisis y probabilidades, del cual se deduce en particular el teorema de convergencia para martingalas. Las ideas 
expuestas en [1] son muy diferentes a las expuestas aquí, y parece más sencillo y natural, en el problema concreto de la convergencia de martingalas con métodos no standard, el recurso a las sucesiones finitas.

\section{Apéndice}

Debido a su importancia ofrezco una demostración completa de los puntos (i), (ii) y (iv) de la proposición 2 recurriendo al axioma del transfer versión IST.

Para cada $\epsilon>0, n, m$ enteros, $n \leq m$, definimos los eventos $A_{n, m}^{\epsilon} y B_{n, m}^{\epsilon}$ :

$$
\begin{aligned}
& A_{n, m}^{\epsilon}=\left\{\max _{n \leq i \leq m}\left|X_{i}-X_{m}\right|>\epsilon\right\}, \\
& B_{n, m}^{\epsilon}=\left\{\max _{n \leq i, j \leq m}\left|X_{i}-X_{j}\right|>\epsilon\right\}
\end{aligned}
$$

(i) La condición implica que para todo $\epsilon>0, P\left(\bigcap_{n \in \mathbb{N}} \bigcup_{m \geq n} A_{n, m}^{\epsilon}\right)=0$. Siendo intersección decreciente de eventos, para todo $\delta>0$ existe $N$ tal que $P\left(\bigcup_{m \geq N} A_{n, m}^{\epsilon}\right)<d$. Si $\epsilon$ es standard la proposición anterior es interna con constantes standard y gracias al transfer podemos suponer que el $\delta$ y el $N$ son también standard. Si $\gamma \leq \mu$ son enteros ilimitados $A_{\gamma, \mu}^{\epsilon} \subset \bigcup_{m \geq N} A_{N, m}^{\epsilon}$ para todo $N$ limitado por lo que $P($ Aeg, $m)<\delta$. Esto es cierto para todo $\delta$ standard positivo, por lo cual $P\left(A_{\gamma, \mu}^{\epsilon}\right) \approx 0$.

(ii) La condición es equivalente a que exista $\mu$ entero ilimitado tal que $P\left(A_{\gamma, \mu}^{\epsilon}\right) \approx 0$ para todo $\epsilon$ standard positivo y $\gamma \leq \mu$ ilimitado. Como $B_{n, m}^{\epsilon} C A e / 4 n, m$ se sigue que $P(B e g, m) \approx 0$. Si $\delta$ es standard positivo la propiedad interna $P\left(B_{n, m}^{\epsilon}\right)<\delta$ se cumple para todo $n$ ilimitado menor que $\mu$ y por "overspill" debe existir $n$ standard que también la cumple. Ahora bien para este $n$, la sucesión de eventos $\left(B_{n, k}^{\epsilon}, k \geq\right.$ $n$ ) es standard y creciente por lo que $P\left(\bigcup_{m \geq n} B_{n, m}^{\epsilon}\right) \approx P\left(B_{n, \mu}^{\epsilon}\right)<\delta$. Siendo $\delta$ standard positivo arbitrario obtenemos $P\left(\bigcap_{n \in \mathbb{N}} \bigcup_{m \geq n} B_{n, m}^{\epsilon}\right) \approx 0$, y es 0 pues el evento considerado es standard. Por transfer esta propiedad se cumple además para todo $\epsilon>0$, de donde se concluye.

(iv) El concepto de equiintegrabilidad de una sucesión de variables aleatorias $\left(X_{n}\right)_{n \in \mathbb{N}}$ significa

$$
\lim _{a \rightarrow \infty} \limsup _{n \rightarrow \infty} E\left(\left|X_{n}\right| 1_{\left|X_{n}\right|>a}\right)=0
$$

Esta se traduce como:

$$
\forall \epsilon>0, \exists K \text { y } N\left(a>K, n>N \Rightarrow E\left(\left|X_{n}\right| 1_{\left|X_{n}\right|>a}\right)<\epsilon\right)
$$

Siendo $X$ standard, podemos aplicar el axioma de transfer y la fórmula anterior es equivalente a:

$\forall$ standard $\epsilon>0, \exists$ standard $k y$ standard $N\left(a>K, n>N \Rightarrow E\left(\left|X_{n}\right| 1_{\left|X_{n}\right|>a}\right)<\epsilon\right)$

Escrito en esta forma deducimos que si $n \approx \infty, a \approx \infty$, se cumple en particular que:

$$
\forall \text { standard } \epsilon>0, E\left(\left|X_{n}\right| 1_{\left|X_{n}\right|>a}\right)<\epsilon
$$

es decir que $E\left(\left|X_{n}\right| 1_{\mid X_{\mid}>a}\right) \approx 0$ que es el resultado buscado. 
SUBMARTINGALAS FINITAS, TEOREMAS DE CONVERGENCIA Y MÉTODOS NO STANDARD23

\section{Referencias}

[1] Bliedtner, J.; Loeb, P. (1992) "A reduction technique for limit theorems in analysis and probability", Ark. Mat., 30(1): 25-43.

[2] Dacunha-Castelle, D.; Duflo, M. (1983) Probabilités et Statistiques, Tomo 2: Problèmes à Temps Mobile. Masson, Paris.

[3] Diener, F.; Reeb, G. (1989) Analyse Non Standard Hermann, Paris.

[4] Doob, J. L. (1953) Stochastic Processes. John Wiley and Sons, New York.

[5] Helms, L.; Loeb, P. (1982) "A nonstandard proof of the martingale convergence theorem", Rocky Mountain Journal of Mathematics, 12(1).

[6] Nelson, E. (1977) "Internal Sey Theory : a new approach to nonstandard analysis", Bulletin of the AMS, 83(6).

[7] Nelson, E. (1987) Radically Eelementary Probability Theory. Princeton University Press.

[8] Neveu, J. (1972) Martingales à Temps Discret. Masson, Paris. 